\title{
Development and characterization of SSR markers from Pinus massoniana and their transferability to $P$. elliottii, $P$. caribaea and P. yunnanensis
}

\author{
Y.H. Feng ${ }^{1,2}$, Z.Q. Yang ${ }^{2}$, J. Wang ${ }^{1}$, Q.F. Luo ${ }^{1,2}$ and H.G. Li ${ }^{1}$ \\ ${ }^{1}$ Key Laboratory of Forest Genetics and Biotechnology, \\ Nanjing Forestry University, Nanjing, Jiangsu, China \\ ${ }^{2}$ Timber Forest Research Division, \\ Guangxi Zhuang Autonomous Region Forestry Research Institute, \\ Nanning, Guangxi, China
}

Corresponding author: H.G. Li

E-mail: hgli@njfu.edu.cn

Genet. Mol. Res. 13 (1): 1508-1513 (2014)

Received December 6, 2012

Accepted June 26, 2013

Published March 12, 2014

DOI http://dx.doi.org/10.4238/2014.March.12.2

\begin{abstract}
Pinus massoniana (Masson's pine) is a widespread tree species in central and southern China and northern Vietnam; it is valued for rosin and paper production. Despite the significant economic value of Masson's pine, little work has been done on its molecular genetics. We developed 318 SSR primers from genome sequences of $P$. massoniana, and we identified 10 polymorphic markers. The
\end{abstract}


number of alleles in the population of $P$. massoniana that we examined ranged from two to four, and the Shannon diversity index ranged from 0.150 to 1.133 . Cross-species transferability of the 318 SSRs was also analyzed in the slash pine (Pinus elliottii), the Caribbean pine (Pinus caribaea) and the Yunnan pine (Pinus yunnanensis); 15, 10, and 10 primer pairs generated polymorphic amplification, respectively. These sets of polymorphic SSR markers will be useful for population genetics studies of $P$. massoniana, for genetic identification of interspecific hybridization, and for phylogeographic studies of Pinus spp.

Key words: Pinus massoniana; SSR; Cross-species transferability

\section{INTRODUCTION}

Masson's pine (Pinus massoniana) is native to a wide area of central and southern China and northern Vietnam, and it is a common tree species in plantation forestry for replacing or compensating for the loss of the natural forest in southern China. The plantations of Masson's pine are estimated to cover 6 million hectares of land, accounting for 15\% of the total Chinese marketed timber (Zhang et al., 2012). With its high cellulose content and long fiber, Masson's pine wood has a multitude of uses, mostly for pulp and paper making, as well as for rosin production (Wilson, 1993; Chen et al., 1996; Cheng et al., 2004). Despite the substantial economic value of Masson's pine, progress in its molecular genetics is very limited. This is mostly attributed to the lack of ideal DNA markers in Masson's pine, even in the genus Pinus.

Undoubtedly, SSRs (single sequence repeats), co-dominant molecular markers, are currently ideal markers that are widely used in population genetics, construction of genetic maps and evolutionary studies of plants (Zietkiewicz et al., 1994; Cuadrado and Schwarzacher; 1998; Gonzalo et al., 2005). Therefore, the development of SSR markers for Masson's pine would provide useful tools to investigate population genetic structure, to construct genetic maps and to implement MAS (marker assistant selection) in this species. Furthermore, the set of SSR markers developed in Masson's pine would also have potential application value in the genetic identification of interspecific hybridization and the phylogeographic study of the genus Pinus. Here, we developed a set of SSRs derived from the genome of Masson's pine and evaluated their transferability in the genus Pinus.

\section{MATERIAL AND METHODS}

\section{Plant material and DNA extraction}

Altogether, 110 trees (genotypes) from four pine species were taken as plant material, of which 30 trees came from Pinus massoniana Lamb (Masson's pine), 30 trees from $P$. elliottii Engelm (slash pine), 30 trees from P. yunnanensis Franch (Yunnan pine), and 20 trees from $P$. caribaea Morelet (Caribbean pine).Young leaves were collected from each tree and stored in a refrigerator. All plant samples above were collected from 
the pine gene pool in Nanning Forestry Research Institute (Nanning, Guangxi, China, $\left.23^{\circ} 10^{\prime} 08^{\prime \prime} \mathrm{N}, 107^{\circ} 59^{\prime} 40^{\prime \prime} \mathrm{E}\right)$. Genomic DNA was extracted from young leaves using the CTAB method (Doyle and Doyle, 1987).

\section{DNA sequencing and SSR identification}

Genomic DNA sequences of Pinus massoniana Lamb were obtained through the Solexa sequencing technology (Cronn et al., 2008; Mardis, 2008). The SSRs were searched from the genome sequences using the SSRIT software (Temnykh et al., 2001). The screening criteria were set for the detection of di-, tri-, tetra-, penta-, and hexanucleotide motifs with a minimum of six, four, three, three, and three repeats, respectively. A total of 736 SSRs were found. SSRs that had low GC content or palindromic sequences in their flanking region were excluded from primer design. The major parameters for primer design were set as follows: primer length from 18 to 22 nucleotides with 20 as the optimum, PCR product size from 200 to $500 \mathrm{bp}$, optimal annealing temperature of $55^{\circ} \mathrm{C}$, and $\mathrm{GC}$ content from 40 to $65 \%$ with $50 \%$ as the optimum. Finally, 311 locus-specific SSR primers were designed using the PRIMER 3 software (Steve and Helen, 2000).

\section{PCR amplification, cross-species transferability and polymorphism analysis}

To assess polymorphism, polymerase chain reaction (PCR) was performed in a $10 \mu \mathrm{L}$ reaction solution containing 10-20 ng genomic DNA, $10 \mathrm{mM}$ Tris- $\mathrm{HCl}, \mathrm{pH}$ 8.0, $50 \mathrm{mM} \mathrm{KCI}$, $2.5 \mathrm{mM} \mathrm{MgCl}, 0.2 \mathrm{mM}$ dNTPs, $0.25 \mu \mathrm{M}$ each primer, and $0.8 \mathrm{U}$ Taq polymerase (Generay, Shanghai, China). The amplification protocol consisted of an initial denaturation at $94^{\circ} \mathrm{C}$ for $4 \mathrm{~min}$, followed by 30 cycles of $15 \mathrm{~s}$ at $94^{\circ} \mathrm{C}, 15 \mathrm{~s}$ at the appropriate annealing temperature and $30 \mathrm{~s}$ at $72^{\circ} \mathrm{C}$, ending with a final extension at $72^{\circ} \mathrm{C}$ for $15 \mathrm{~min}$. Amplified products were separated on $8 \%$ denaturing polyacrylamide gels and visualized by silver staining. A 50 -bp DNA ladder was used to identify alleles. The number of alleles $\left(N_{A}\right)$ and Shannon's information index $(I)$ were performed using Popgene1.32 (Yeh et al.,1999).

\section{RESULTS AND DISCUSSION}

Of the 318 SSR markers tested, 305 generated expected amplification products, and 10 of them were polymorphic. The genetic variability of the 10 polymorphic markers was estimated by genotyping thirty individuals of Masson's pine. Population genetic parameters were calculated through the PopGene32 software (Yeh et al., 1999). The number of alleles per locus ranged from two to four, with an average value of 2.4. The Shannon diversity index $(I)$ (Lewontin, 1972) was from 0.150 to 1.133 , with an average of 0.503 (Table 1).

To test cross-species transferablity, the same set of 318 SSR markers were amplified in thirty individuals of slash pine, twenty individuals of Caribbean pine, and thirty individuals of Yunnan pine. Thirty-four polymorphic SSR markers were characterized: 15 markers in slash pine, 10 markers in Caribbean pine, and 10 markers in Yunnan pine. The number of alleles per locus ranged from two to four, and the Shannon diversity index ( $I$ ) per locus was from 0.087 to 1.144 (Table 1). The characteristics of 34 polymorphic SSR markers in Pinus are listed in Table 2. 
This set of polymorphic SSR markers may serve as powerful tools not only for the identification of genetic structure and gene flow, the construction of genetic maps, and MAS in Masson's pine, but also for the identification of interspecific hybrids and phylogeographic studies in the genus Pinus.

Table 1. Results of cross-species amplification of thirty-four polymorphic SSR markers in Pinus.

\begin{tabular}{|c|c|c|c|c|}
\hline Locus & Species & Sample size & $N_{\mathrm{A}}$ & I \\
\hline PF322 & Masson pine & 30 & 2 & 0.678 \\
\hline PF402 & Masson pine & 30 & 3 & 0.66 \\
\hline PF408 & Masson pine & 30 & 3 & 0.581 \\
\hline PF460 & Masson pine & 30 & 2 & 0.572 \\
\hline PF463 & Masson pine & 30 & 4 & 1.133 \\
\hline PF464 & Masson pine & 30 & 2 & 0.15 \\
\hline PF492 & Masson pine & 30 & 2 & 0.604 \\
\hline PF569 & Masson pine & 30 & 2 & 0.251 \\
\hline PF576 & Masson pine & 30 & 2 & 0.204 \\
\hline PF615 & Masson pine & 30 & 2 & 0.199 \\
\hline PF310 & Slash pine & 30 & 2 & 0.2411 \\
\hline PF314 & Slash pine & 30 & 2 & 0.2868 \\
\hline PF377 & Slash pine & 30 & 3 & 0.7216 \\
\hline PF383 & Slash pine & 30 & 3 & 0.8647 \\
\hline PF402 & Slash pine & 30 & 3 & 0.3368 \\
\hline PF429 & Slash pine & 30 & 4 & 1.1439 \\
\hline PF489 & Slash pine & 30 & 4 & 1.0678 \\
\hline PF494 & Slash pine & 30 & 2 & 0.6474 \\
\hline PF505 & Slash pine & 30 & 4 & 0.7535 \\
\hline PF511 & Slash pine & 30 & 3 & 0.3887 \\
\hline PF549 & Slash pine & 30 & 2 & 0.5961 \\
\hline PF552 & Slash pine & 30 & 3 & 0.5524 \\
\hline PF585 & Slash pine & 30 & 3 & 0.5838 \\
\hline PF593 & Slash pine & 30 & 4 & 0.9941 \\
\hline PF615 & Slash pine & 30 & 2 & 0.5004 \\
\hline PF429 & Caribbean pine & 20 & 4 & 1.054 \\
\hline PF431 & Caribbean pine & 20 & 2 & 0.5 \\
\hline PF436 & Caribbean pine & 20 & 2 & 0.631 \\
\hline PF441 & Caribbean pine & 20 & 2 & 0.418 \\
\hline PF443 & Caribbean pine & 20 & 2 & 0.588 \\
\hline PF489 & Caribbean pine & 20 & 2 & 0.693 \\
\hline PF494 & Caribbean pine & 20 & 2 & 0.423 \\
\hline PF511 & Caribbean pine & 20 & 3 & 0.88 \\
\hline PF533 & Caribbean pine & 20 & 2 & 0.647 \\
\hline PF606 & Caribbean pine & 20 & 2 & 0.562 \\
\hline PF334 & Yunnan pine & 30 & 2 & 0.3251 \\
\hline PF403 & Yunnan pine & 30 & 2 & 0.5716 \\
\hline PF408 & Yunnan pine & 30 & 2 & 0.6109 \\
\hline PF463 & Yunnan pine & 30 & 2 & 0.0871 \\
\hline PF464 & Yunnan pine & 30 & 2 & 0.2573 \\
\hline PF492 & Yunnan pine & 30 & 2 & 0.5269 \\
\hline PF555 & Yunnan pine & 30 & 2 & 0.3326 \\
\hline PF557 & Yunnan pine & 30 & 2 & 0.2937 \\
\hline PF561 & Yunnan pine & 30 & 2 & 0.251 \\
\hline PF615 & Yunnan pine & 30 & 2 & 0.6906 \\
\hline
\end{tabular}

$N_{\mathrm{A}}=$ number of alleles; I = shannon's information index. 
Table 2. Characteristics of thirty-four microsatellite primers developed in Pinus.

\begin{tabular}{|c|c|c|c|c|}
\hline Locus & Primer sequence $\left(5^{\prime}-3^{\prime}\right)$ & Repeat motif & Expected size (bp) & $\mathrm{Ta}\left({ }^{\circ} \mathrm{C}\right)$ \\
\hline PF310 & $\begin{array}{l}\text { F: CGTCCCTCCCGTTTATTG } \\
\text { R: GGTGACCTTGCTGCCTTG }\end{array}$ & $(\text { TTTG })_{4}$ & 356 & 56 \\
\hline PF314 & $\begin{array}{l}\text { F: ATGCTTGCCTTATGACTTGACA } \\
\text { R: CAGCACTACTATTGCAGGGAGA }\end{array}$ & $(\mathrm{CCAAA})_{3}$ & 336 & 57 \\
\hline PF322 & $\begin{array}{l}\text { F: CTGTGGCTATCTTTGACTCTGC } \\
\text { R: GAAATTCTTGTGGTCGGATGTA }\end{array}$ & $(\mathrm{ACCT})_{4}$ & 411 & 57 \\
\hline PF334 & $\begin{array}{l}\text { F: GGGTGTATAGAGGGAAGGATTT } \\
\text { R: ACAGAGGGGCTAGGTCAGG }\end{array}$ & $(\mathrm{TTGC})_{4}$ & 383 & 56 \\
\hline PF377 & $\begin{array}{l}\text { F: TGTTTCACCCACGCCAGTC } \\
\text { R: GCCATTTCAAAAGAGGCAGAT }\end{array}$ & $(\mathrm{TAA})_{5}$ & 434 & 58 \\
\hline PF383 & $\begin{array}{l}\text { F: TGGGCGTAGGAGGGTTGT } \\
\text { R: GGGCTTTCTTTGTGCTATTGG }\end{array}$ & $(\mathrm{AGTC})_{5}$ & 241 & 58 \\
\hline PF402 & $\begin{array}{l}\text { F: ATGCTCATAATGAAATGGGACT } \\
\text { R: ATGCATTGCACTGCACGT }\end{array}$ & $(\mathrm{TGTCAG})_{3}$ & 203 & 55 \\
\hline PF403 & $\begin{array}{l}\text { F: ACGATTCTTGCCAACGCT } \\
\text { R: GCTGGAACAATTCAAATTTTGT }\end{array}$ & $(\text { AAATTG })_{3}$ & 314 & 56 \\
\hline PF408 & $\begin{array}{l}\text { F: TACAAAGGACTCCAGCAAAGTG } \\
\text { R: GCGGATGTGCGAGGTTATG }\end{array}$ & $(\text { TGAAT })_{3}$ & 302 & 57 \\
\hline PF429 & $\begin{array}{l}\text { F: GCTCCATGTTTGGAAGGG } \\
\text { R: CCAGCCAGCGATCTAAGTAA }\end{array}$ & $(\text { TTGC) })_{3}$ & 401 & 55 \\
\hline PF431 & $\begin{array}{l}\text { F: TCCACATCTATGGGTGCTTG } \\
\text { R: GCTTCATTGCTGAAAGGTCA }\end{array}$ & $(\mathrm{TCA})_{4}$ & 247 & 56 \\
\hline PF436 & $\begin{array}{l}\text { F: CAGGGAAGGAGACAAAACA } \\
\text { R: GAGGGAAGAAGAAAGACATAAA }\end{array}$ & $(\mathrm{CCTC})_{3}$ & 207 & 52 \\
\hline PF441 & $\begin{array}{l}\text { F: CCACCAATGACATCAAGGAG } \\
\text { R: TTACGAGTAAGCAAGTGACAGC }\end{array}$ & $(\mathrm{CAAT})_{3}$ & 324 & 55 \\
\hline PF443 & $\begin{array}{l}\text { F: TTTCCTTATCGCCCAAGT } \\
\text { R: GGCCCAATGATTATCATACA }\end{array}$ & $(\mathrm{ATTT})_{3}$ & 295 & 52 \\
\hline PF460 & $\begin{array}{l}\text { F: AACCTCATCTGAAGAAGCCATA } \\
\text { R: AGCAGCATTACCAGCAACATA }\end{array}$ & $(\mathrm{TGC})_{4}$ & 325 & 56 \\
\hline PF463 & $\begin{array}{l}\text { F: CTCTGGGTCCGTACTATCCG } \\
\text { R: GAAGCAAAGGCGAGCAAA }\end{array}$ & $(\mathrm{ATCT})_{3}$ & 253 & 57 \\
\hline PF464 & $\begin{array}{l}\text { F: TTGCTCGCCTTTGCTTCT } \\
\text { R: GCCTCCTTTACCACAGCCT }\end{array}$ & $(\mathrm{ATGC})_{3}$ & 233 & 56 \\
\hline PF489 & $\begin{array}{l}\text { F: GGGAAGCGAAAGTGATTATTG } \\
\text { R: CCTGGGACTGAGACTGATTGA }\end{array}$ & $(\mathrm{AATA})_{3}$ & 446 & 57 \\
\hline PF492 & $\begin{array}{l}\text { F: TTATGTTGCGGATCAAGAATT } \\
\text { R: ACATGGGCACAACTTGCTAT }\end{array}$ & $(\mathrm{GA})_{7}$ & 238 & 55 \\
\hline PF494 & $\begin{array}{l}\text { F: GCCCTTAATGGATTATTCTGC } \\
\text { R: TTTCTACGCCTCCTCCTGTC }\end{array}$ & $(\mathrm{AGG})_{4}$ & 447 & 56 \\
\hline PF505 & $\begin{array}{l}\text { F: AGAGGAATAAGGTAAGGGATGG } \\
\text { R: GAAAACCGCTTCAATGGC }\end{array}$ & $(\text { TTAT })_{3}$ & 416 & 56 \\
\hline PF511 & $\begin{array}{l}\text { F: TCCTTCTGTTATTGTACCCTCC } \\
\text { R: GATTGATTGTATTGCACCCAC }\end{array}$ & $(\mathrm{CAGG})_{3}$ & 372 & 55 \\
\hline PF533 & $\begin{array}{l}\text { F: TCCCGAAGAAAGGAACAC } \\
\text { R: CATAGAACGCACGCAAAT }\end{array}$ & $(\mathrm{AAGC})_{3}$ & 224 & 52 \\
\hline PF549 & $\begin{array}{l}\text { F: GGGGTCAGGTTTGGCATC } \\
\text { R: CGGGCTAAGCTAAGCAGGTA }\end{array}$ & $(\mathrm{CTGA})_{3}$ & 215 & 57 \\
\hline PF552 & $\begin{array}{l}\text { F: TGGTTTGGACATGGACTCAC } \\
\text { R: AGATTTCCTTCAGAGGTTTCG }\end{array}$ & $(\mathrm{ATC})_{4}$ & 220 & 55 \\
\hline PF555 & $\begin{array}{l}\text { F: GGTGAGGGTAGTCGTCTGTCT } \\
\text { R: CGATGAAGGGCAACTATGAT }\end{array}$ & $(\mathrm{CCTT})_{3}$ & 377 & 55 \\
\hline PF557 & $\begin{array}{l}\text { F: AGCACTCATCAACTTTCCAGC } \\
\text { R: AAGATTAGATAAGTCCCGTCCC }\end{array}$ & $(\mathrm{AATG})_{3}$ & 440 & 56 \\
\hline PF561 & $\begin{array}{l}\text { F: TTATTGGGCAGGAGGACG } \\
\text { R: TGGTTGCTTTAGATCGAACAG }\end{array}$ & $(\mathrm{GAAC})_{3}$ & 327 & 55 \\
\hline PF569 & $\begin{array}{l}\text { F: AACAGAAAGGAATCAAGTAGGC } \\
\text { R: AATCACATGAAATGCTGGAAA }\end{array}$ & $(\mathrm{AGTA})_{3}$ & 308 & 55 \\
\hline PF576 & $\begin{array}{l}\text { F: CTTGGCGGCACTTATTGA } \\
\text { R: ATGATATGATGGGGCTGGTA }\end{array}$ & $(\mathrm{CTTA})_{3}$ & 211 & 55 \\
\hline PF585 & $\begin{array}{l}\text { F: CTCCCGCTTTTCCTCCAC } \\
\text { R: CCGTTTTCATTTCAGTCCTTG }\end{array}$ & $(\mathrm{CACT})_{3}$ & 338 & 57 \\
\hline
\end{tabular}

Continued on next page 
Table 2. Characteristics of thirty-four microsatellite primers developed in Pinus.

\begin{tabular}{|c|c|c|c|c|}
\hline Locus & Primer sequence $\left(5^{\prime}-3^{\prime}\right)$ & Repeat motif & Expected size (bp) & $\mathrm{Ta}\left({ }^{\circ} \mathrm{C}\right)$ \\
\hline$\overline{\text { PF593 }}$ & $\begin{array}{l}\text { F: AACTCCCCTTCCCCATACG } \\
\text { R: ATTCCCGCCGACTCCTAA }\end{array}$ & $(\mathrm{CTTG})_{3}$ & 427 & 57 \\
\hline PF606 & $\begin{array}{l}\text { F: CAAGCGGAGTATGTCAGGTAG } \\
\text { R: ATGTTTGTAGGTCGTTAGAGGG }\end{array}$ & $(\mathrm{AT})_{6}$ & 407 & 55 \\
\hline PF615 & $\begin{array}{l}\text { F: TAAATGATTGGCTATCGGAGAC } \\
\text { R: CCCGCTCTGAAGATGTTGTC }\end{array}$ & $\begin{array}{c}(\mathrm{ATTGAG})_{4} \\
(\mathrm{ACTA})_{3}(\mathrm{ACTC})_{3}\end{array}$ & 399 & 56 \\
\hline
\end{tabular}

$\mathrm{Ta}=$ annealing temperature.

\section{ACKNOWLEDGMENTS}

Research supported by the BaGui-Scholar Foundation, Guangxi Provincial Scientific and Technical Research Project (\#GKG1123004-4A), Jiangsu Provincial Graduate Student Innovation Program (\#CXZZ12_0541), the Program Development of Jiangsu Higher Education Institutions (PAPD), and State Financial Project for the Commercialization of Scientific \& Technical Achievements (\#2011TK090). We are grateful to Y.L. Huang for his assistance in plant sample collection. We also thank L.S. Li for genomic DNA sequencing and D.S. Wu for technical support.

\section{REFERENCES}

Chen TH, Wang ZR and Xu L (1996). Chem. Industry Forest Prod. Genetic variation of wood properties in Pinus massoniana Lamb and the utilization potential in papermaking industry. Chem. Industry Forest Prod. 16: 71-78.

Cheng XS, Chen WJ, Chen YP, Chen YX, et al. (2004). Preparation and Properties of HBS Lignin from Masson Pine. Chem. Res. Chinese Univ. 20: 225-228.

Cronn R, Liston A, Parks M, Gernandt DS, et al. (2008). Multiplex sequencing of plant chloroplast genomes using Solexa sequencing-by-synthesis technology. Nucleic Acids Res. 36: e122.

Cuadrado A and Schwarzacher T (1998). The chromosomal organization of simple sequence repeats in wheat and rye genomes. Chromosoma 107: 587-594.

Doyle JJ and Doyle JL (1987). Isolation of plant DNA from fresh tissue. Focus 12: 13-15.

Gonzalo MJ, Oliver M, Garcia-Mas J, Monfort A, et al. (2005). Simple-sequence repeat markers used in merging linkage maps of melon (Cucumis melo L.). Theor. Appl. Genet. 110: 802-811.

Lewontin RC (1972). The apportionment of human diversity. Evolutionary Biol. 6: 381-398.

Mardis ER (2008). The impact of next-generation sequencing technology on genetics. Trends Genet. 24: 133-141.

Steve R and Helen S (2000). Primer3 on the WWW for General Users and for Biologist Programmers. In: Bioinformatics Methods and Protocols: Methods in Molecular Biology. (Krawetz S and Misener S, eds.). Humana Press, Totowa, 365-386.

Temnykh S, DeClerck G, Lukashova A, Lipovich L, et al. (2001). Computational and experimental analysis of microsatellites in rice (Oryza sativa L.): frequency, length variation, transposon associations, and genetic marker potential. Genome Res. 11: 1441-1452.

Wilson LF (1993). China's masson pine forests: cure or curse. J. Forestry 91: 30-33.

Yeh FC, Yang RC and Boyle TBJ (1999). POPGENE version 1.32, Microsoft Window- Based Free Ware for Population Genetic Analysis. Computer Program and Documentation Distributed by University of Alberta and Centre for International Forestry Research, Alberta.

ZhangY, Yang Q, Zhou ZC and Jin GQ (2012). Divergence among masson pine parents revealed by geographical origins and SSR markers and their relationships with progeny performance. New Forests 44: 341-355.

Zietkiewicz E, Rafalski A and Labuda D (1994). Genome fingerprinting by simple sequence repeat (SSR)-anchored polymerase chain reaction amplification. Genomics 20: 176-183. 